\title{
Erratum to: (Hyper)polarizability density analysis for open-shell molecular systems based on natural orbitals and occupation numbers
}

\author{
Masayoshi Nakano - Hitoshi Fukui - Takuya Minami $\cdot$ Kyohei Yoneda \\ Yasuteru Shigeta · Ryohei Kishi • Benoît Champagne · Edith Botek • \\ Takashi Kubo $\cdot$ Koji Ohta $\cdot$ Kenji Kamada
}

Published online: 2 November 2011

(C) Springer-Verlag 2011

\section{Erratum to: Theor Chem Acc \\ DOI 10.1007/s00214-010-0871-y}

Due to publisher's error that occurred at the typesetting stage and which was not corrected at the proof stage, reference 81 was inadvertently deleted with the result that references $81-104$ should be $82-105$, with reference 81 being

Bulat FA, Toro-Labbe A, Champagne B, Kirtman B, Yang W (2005) J Chem Phys 123:014319

The online version of the original article can be found under doi:10.1007/s00214-010-0871-y.

M. Nakano $(\varangle) \cdot$ H. Fukui · T. Minami · K. Yoneda .

Y. Shigeta $\cdot$ R. Kishi

Department of Materials Engineering Science, Graduate School

of Engineering Science, Osaka University, Toyonaka,

Osaka 560-8531, Japan

e-mail: mnaka@cheng.es.osaka-u.ac.jp

B. Champagne $\cdot$ E. Botek

Laboratoire de Chimie Théorique, Facultés Universitaires

Notre-Dame de la Paix (FUNDP), rue de Bruxelles, 61,

5000 Namur, Belgium

e-mail: benoit.champagne@fundp.ac.be

T. Kubo

Department of Chemistry, Graduate School of Science,

Osaka University, Toyonaka, Osaka 560-0043, Japan

e-mail: kubo@chem.sci.osaka-u.ac.jp

\section{K. Ohta $\cdot$ K. Kamada}

Research Institute for Ubiquitous Energy Devices,

National Institute of Advanced Industrial Science

and Technology (AIST), Ikeda, Osaka 563-8577, Japan

e-mail: k.ohta@aist.go.jp

K. Kamada

e-mail: k.kamada@aist.go.jp
The only reference in the text that then needs to be changed is reference 104 (above Sect. 5 Concluding remarks) which should then be reference 105 . The corrected line is given below:

These properties will be reintroduced well by the complete active space (CAS)-DFT method [94-105].

The amended list of references $81-105$ are as follows:

81. Bulat FA, Toro-Labbé A, Champagne B, Kirtman B, Yang W (2005) J Chem Phys 123:014319

82. Mori-Sanchez P, Wu Q, Yang W (2003) J Chem Phys 119:11031

83. Sekino H, Maeda Y, Kamiya M, Hirao K (2007) J Chem Phys 126:014107

84. Kirtman B, Bonness S, Ramirez-Solis A, Champagne B, Matsumoto H, Sekino H (2008) J Chem Phys 128:114108

85. Bonness S, Fukui H, Yoneda K, Kishi R, Champagne B, Botek E, Nakano M (2010) Chem Phys Lett 493:195

86. Nakano M, Takebe A, Kishi R, Ohta S, Nate M, Kubo T, Kamada K, Ohta K, Champagne B, Botek E, Takahashi H, Furukawa S, Morita S, Nakasuji K (2006) Chem Phys Lett 432:473

87. Song J-W, Hirosawa T, Tsuneda T, Hirao K (2007) J Chem Phys 126:154105

88. Frisch MJ, Trucks GW, Schlegel HB, Scuseria GE, Robb MA, Cheeseman JR, Scalmani G, Barone V, Mennucci B, Petersson GA, Nakatsuji H, Caricato M, Li X, Hratchian HP, Izmaylov AF, Bloino J, Zheng G, Sonnenberg JL, Hada M, Ehara M, Toyota K, Fukuda R, Hasegawa J, Ishida M, Nakajima T, Honda Y, Kitao O, Nakai H, Vreven T, Montgomery JA Jr, Peralta JE, Ogliaro F, Bearpark M, Heyd JJ, Brothers E, Kudin KN, Staroverov VN, Kobayashi R, 
Normand J, Raghavachari K, Rendell A, Burant JC, Iyengar SS, Tomasi J, Cossi M, Rega N, Millam NJ, Klene M, Knox JE, Cross JB, Bakken V, Adamo C, Jaramillo J, Gomperts R, Stratmann RE, Yazyev O, Austin AJ, Cammi R, Pomelli C, Ochterski JW, Martin RL, Morokuma K, Zakrzewski VG, Voth GA, Salvador P, Dannenberg JJ, Dapprich S, Daniels AD, Farkas Ö, Foresman JB, Ortiz JV, Cioslowski J, Fox DJ (2009) Gaussian 09, Revision A.1, Gaussian, Inc., Wallingford CT

89. Filatov M, Cremer D (2005) J Chem Phys 123:124101

90. Nakano M, Yamada S, Shigemoto I, Yamaguchi K (1996) Chem Phys Lett 250:247

91. Nakano M, Yamaguchi K (1994) Phys Rev A 50:2989

92. Nakano M, Yamaguchi K, Matsuzaki Y, Tanaka K, Yamabe T (1995) J Chem Phys 102:2986

93. Yanai T, Tew DP, Handy NC (2004) Chem Phys Lett 393:51

94. Savin A, Flad HJ (1995) Int J Quantum Chem 56:327
95. Gori-Giorgi P, Savin A (2006) Phys Rev A 73:3250

96. Fromager E, Toulouse J, Jensen HJA (2007) J Chem Phys 126:074111

97. Kusakabe K (2001) J Phys Soc Jpn 70:2038

98. Yamanaka S, Nakata K, Takada T, Kusakabe K, Ugalde JM, Yamaguchi K (2006) Chem Lett 35:242

99. Yamanaka S, Nakata K, Ukai T, Takada T, Yamaguchi K (2006) Int J Quantum Chem 16:3312

100. Miehlich B, Stoll H, Savin A (1997) Mol Phys 91:527

101. Gräfenstein J, Cremer D (2000) Chem Phys Lett 316:569

102. Gusarov S, Malmqvist PA, Lindh R, Roos BO (2004) Theor Chem Acc 112:84

103. Pérez-Jiménez, AJ, Pérez-Jord JM (2007) Phys Rev A 75:012503

104. Takeda R, Yamanaka S, Yamaguchi K (2002) Chem Phys Lett 366:321

105. Takeda R, Yamanaka S, Yamaguchi K (2004) Int J Quantum Chem 96:463 